\title{
DESENVOLVIMENTO E VALIDAÇÃO DE METODOLOGIA ANALÍTICA POR ESPECTROFOTOMETRIA NO VISÍVEL PARA QUANTIFICAÇÃO DE TANINOS TOTAIS NA CASCA DO CAULE DE Simarouba amara Aubl. ${ }^{1}$
}

Iasmine Andreza Basilio dos Santos Alves², Henrique Marcelino Miranda ${ }^{3}$, Antenor Pereira Barbosa ${ }^{4}$, Karina Perreli Randau ${ }^{5}$ e Luiz Alberto Lira Soares ${ }^{5}$

RESUMO - As cascas do caule de Simarouba amara Aubl., Simaroubaceae, são amplamente utilizadas pela medicina popular brasileira e de vários países das Américas Central e do Sul para o tratamento de malária, parasitas intestinais, diarreia, anemia e febre. Apesar de os quassinoides serem considerados os marcadores quimiotaxonômicos da espécie, a indisponibilidade de padrões para comercialização tem sido um dos grandes obstáculos à realização de seu controle de qualidade. Diante disso, o propósito deste estudo foi o desenvolvimento e validação de metodologia analítica por espectrofotometria para a quantificação do teor de taninos totais das cascas do caule de S. amara, visto que esta classe de metabólitos é uma das mais abundantes e representativas já descritas para a espécie. O método proposto baseou-se na quantificação a $760 \mathrm{~nm}$, após a adição do reagente Folin-Ciocalteu, e sua validação incluiu todos os parâmetros preconizados pela RE 899. Os resultados indicaram que o método proposto foi adequadamente desenvolvido e validado, constituindo ferramenta analítica útil para o controle de qualidade de S. amara.

Palavras-chave: Reagente Folin-Ciocalteau; Simaroubaceae; Teor de Taninos.

\section{DEVELOPMENT AND VALIDATION OF ANALYTICAL METHODOLOGY BY SPECTROPHOTOMETRY IN THE VISIBLE FOR QUANTIFICATION OF TOTAL TANNINS IN THE STEM BARK OF Simarouba amara Aubl.}

\begin{abstract}
The stem bark of Simarouba amara Aubl., Simaroubaceae, is widely used in Brazilian folk medicine and in many countries of Central and South America for the treatment of malaria, intestinal parasites, diarrhea, anemia and fever. Although quassinoids are considered chemotaxonomic markers of the species, the unavailability of commercialization standards stay as a challenge for its quality control. Therefore, the purpose of this study was the development and validation of an analytical methodology by spectrophotometry to quantify the total tannin content from stem bark of S. amara, since this class of metabolites is one of the most abundant and representative in the species. The proposed method was based on quantification at $760 \mathrm{~nm}$ after addition of Folin-Ciocalteu reagent and its validation included all parameters recommended by the RE 899. The results showed that the proposed method was adequately developed and validated, and it is an useful analytical tool for the quality control of S. amara.
\end{abstract}

Keywords: Folin-Ciocalteu reagent; Simaroubaceae; Tannin content.

\footnotetext{
${ }^{1}$ Recebido em 26.06.2014 aceito para publicação em 19.11.2014.

${ }^{2}$ Universidade Federal de Pernambuco, Programa de Pós-Graduação em Ciências Farmacêuticas, Recife, Pernambuco - Brasil. E-mail:<iasmine_alves@hotmail.com>.

${ }^{3}$ Universidade Federal de Pernambuco, Graduando em Farmácia, Recife, Pernambuco - Brasil. E-mail: $<$ hmn2206@hotmail.com>.

${ }^{4}$ Instituto Nacional de Pesquisas da Amazônia, Manaus, Amazonas - Brasil. E-mail: <antenor@inpa.gov.br>.

${ }^{5}$ Universidade Federal de Pernambuco, Departamento de Farmácia, Recife, Pernambuco - Brasil. E-mail: $<$ krandau@hotmail.com> e $<$ lals@gmx.de $>$.
} 


\section{INTRODUÇÃO}

A família Simaroubaceae apresenta distribuição pantropical e é caracterizada pela presença de quassinoides, produtos de degradação de triterpenos altamente oxigenados e estruturalmente complexos, os quais consistem em marcadores quimiotaxonômicos dessa família (ALMEIDA et al., 2007). Suas espécies são amplamente utilizadas na medicina popular por apresentarem atividade antimalárica, anti-helmíntica, antitumoral, anti-inflamatória, antiviral, anorexígena, tônica, inseticida e amebicida (ARRIAGA et al., 2002; MUHAMMAD et al., 2004; SARAIVA; PINTO, 2006; CACHET et al., 2009; SILVA et al., 2010). Entre suas atividades farmacológicas comprovadas, destacamse as ações antitumoral in vitro (OGURA et al., 1977), antileucêmica (GHOSH et al., 1977; POLONSKY et al., 1978; VALERIOTE et al., 1998), antiviral in vitro contra herpes vírus, influenza vírus, poliovírus e vírus vaccínia (KAIJ-A-KAMB, 1992), amebicida (WRIGHT et al., 1988) e antimicrobiana contra Salmonella typhosa, Shigella flexneri (CACERES et al., 1990), Plasmodium falciparum (KAUR et al., 2009) e Plasmodium berghei (MARIATH et al., 2009).

Simarouba amara Aubl. é uma árvore tropical de médio porte (cerca de $35 \mathrm{~m}$ ), fanerófita, útil no processo de recuperação de áreas degradadas, encontrada nas Américas Central e do Sul, bem como nas ilhas do Caribe (LORENZI, 2002; DECLERCQ, 2004; NERI et al., 2011). No Brasil, está presente na Amazônia e nos Estados do Maranhão, Ceará, de Pernambuco (LOUREIRO et al., 1979), da Bahia, do Espírito Santo e Rio de Janeiro (LORENZI, 2002; PIRANI; THOMAS, 2013). Na medicina tradicional brasileira, é utilizada no tratamento de anemia, febre, diarreia, disenteria, parasitas intestinais e dispepsia (LORENZI; MATOS, 2002; AMARAL et al., 2006; SCOLES et al., 2006; RIBEIRO et al., 2007; SILVA; FRANCO, 2010). Quanto à constituição química, investigações prévias resultaram no isolamento de alcaloides (LASSAK et al., 1977), quassinoides, triterpenos (POLONSKY et al., 1976; GROSVENOR et al., 2006) e polifenóis (GOVINDARAJU et al., 2009).

Apesar de S. amara apresentar várias atividades farmacológicas já comprovadas, ser extensamente utilizada pela medicina popular do Brasil, ter feito parte da primeira e segunda edições da Farmacopeia Brasileira e constituir notável potencial para pesquisas científicas, ainda não foram desenvolvidos e validados procedimentos analíticos para seu controle de qualidade. A importância do preenchimento dessa lacuna é reforçada pela Agência Nacional de Vigilância Sanitária (ANVISA), por meio da Resolução $n^{\circ} 26 / 2014$, que normatiza o registro de fitoterápicos no Brasil e exige que o controle de qualidade seja realizado em todas as etapas de processamento da droga vegetal, incluindo a análise da matéria-prima (BRASIL, 2014).

Nesse contexto, destacam-se as metodologias espectrofotométricas pelo Ultravioleta-Visível, as quais são de baixo custo, fáceis de realizar, rápidas e aplicáveis ao uso de rotina laboratorial (FERNANDES et al., 2012; MARQUES et al., 2013). Os metabólitos secundários escolhidos para serem determinados em S. amara foram os taninos, visto que esta classe de compostos é abundante e representativa na referida espécie, conforme verificado em triagem fitoquímica preliminar. Para sua quantificação, o método de Folin-Ciocalteu é amplamente empregado (FOLIN; CIOCALTEU, 1927; MONTEIRO et al., 2005; GALLICE et al., 2011). Ele se baseia na redução dos ácidos fosfotúngstico $\left(\mathrm{H}_{3} \mathrm{PW}_{12} \mathrm{O}_{40}\right)$ e fosfomolíbdico $\left(\mathrm{H}_{3} \mathrm{PMo}_{12} \mathrm{O}_{40}\right)$, presentes no reagente, pelos compostos fenólicos presentes na amostra na forma de óxido de tungstênio $\left(\mathrm{W}_{8} \mathrm{O}_{23}\right)$ e óxido de molibdênio $\left(\mathrm{Mo}_{8} \mathrm{O}_{23}\right)$ em meio alcalino. Os óxidos formados apresentam coloração azulada, sendo possível a quantificação da absorvância da solução na região do visível (760 nm). Visto que esse método tem sido considerado eficiente para quantificação de substâncias em várias espécies arbóreas (LOPEZ et al., 2005; MONTEIRO et al., 2005), optou-se por sua escolha para determinar os taninos totais na casca do caule da matéria-prima vegetal.

Diante do exposto, este trabalho objetivou desenvolver e validar uma metodologia espectrofotométrica na faixa visível para determinação de taninos totais na casca do caule de $S$. amara, através do método de Folin-Ciocalteu, a qual possa contribuir para o seu controle de qualidade.

\section{MATERIAL E MÉTODOS}

\subsection{Material Vegetal}

As cascas do caule de S. amara Aubl. foram coletadas na Estação Experimental de Silvicultura Tropical (EEST) do Instituto Nacional de Pesquisas da Amazônia (INPA), situada na BR-174, km 43, Município de Manaus, Amazonas. A exsicata foi depositada no herbário do

Revista Árvore, Viçosa-MG, v.39, n.1, p.37-47, 2015

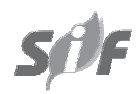


INPA, sob o número 181.975. Após a coleta, o material vegetal foi submetido à secagem à temperatura ambiente e, em seguida, pulverizado em triturador industrial.

\subsection{Reagentes e Substância de Referência}

Todos os reagentes utilizados foram de grau analítico: água destilada, reagente Folin-Ciocalteu (Dinâmica $\left.{ }^{\circledR}\right)$, carbonato de sódio anidro (Synth $\left.{ }^{\circledR}\right)$, pó de pele, polivinilpirrolidona (Basf®) e caseína (Merck $($ ) Pirogalol (Fluka ${ }^{\circledR}$, Pureza e" 98,0\%) foi utilizado como padrão.

\subsection{Determinação Espectrofotométrica de Taninos Totais na Casca de Simarouba amara}

\subsubsection{Preparo da Solução Extrativa}

$1,5000 \mathrm{~g}$ da droga vegetal foram pesados e transferidos para balão com boca esmerilhada de 250 $\mathrm{mL}$ (Falta pedaço de texto). Adicionaram-se $150 \mathrm{~mL}$ de água destilada, que foram levados para aquecimento em banho-maria, sob refluxo, por $30 \mathrm{~min}$, a $60^{\circ} \mathrm{C}$. Em seguida, o balão foi resfriado em água corrente e a solução, transferida para balão volumétrico de $250 \mathrm{~mL}$, com subsequentes lavagens até a remoção total do conteúdo de droga vegetal. Completou-se o volume com água destilada e, após a decantação, o sobrenadante foi filtrado em papel-filtro. Os primeiros $50 \mathrm{~mL}$ foram desprezados.

\subsubsection{Preparo da Solução para Determinação de Polifenois Totais}

Em balão volumétrico de $25 \mathrm{~mL}$, foram adicionados 4,0 mL da solução extrativa, 3,0 mL do reagente FolinCiocaulteu e 10,0 mL de água destilada. O volume final foi completado com a solução de carbonato de sódio $\left(\mathrm{Na}_{2} \mathrm{CO}_{3}\right)$ a $10,75 \%(\mathrm{~m} / \mathrm{v})$. Após $30 \mathrm{~min}$, a absorvância foi mensurada em um comprimento de onda de $760 \mathrm{~nm}$, em espectrofotômetro (EVOLUTION 60S, Thermo Fischer Scientific () , utilizando água destilada como solução de compensação. Todas as operações foram protegidas da luz.

\subsubsection{Preparo da Solução para Polifenóis Não Adsorvidos por Pó de Pele}

Em erlenmeyer de $125 \mathrm{~mL}$ foram adicionados 10,0 mL da solução extrativa e 150,0 mg de pó de pele, os quais foram submetidos à agitação mecânica por 60 min e, em seguida, filtrados em papel-filtro. Alíquota de 4,0 $\mathrm{mL}$ foi retirada do filtrado e transferida, volumetricamente, para balão volumétrico de $25 \mathrm{~mL}$. Em seguida, foram adicionados $10,0 \mathrm{~mL}$ de água destilada, 3,0 mL do reagente Folin-Ciocalteu, sendo o volume completado com solução de carbonato de sódio $\left(\mathrm{Na}_{2} \mathrm{CO}_{3}\right)$ a 10,75\% (m/v). Após $30 \mathrm{~min}$, a absorvância foi lida em um comprimento de onda de $760 \mathrm{~nm}$, utilizando-se água destilada como solução de compensação. Todas as operações foram protegidas da luz.

\subsubsection{Preparo da Solução-Padrão de Pirogalol}

Pirogalol foi escolhido como padrão por já ser utilizado para quantificação de taninos na Farmacopeia Brasileira $-5^{\text {a }}$ edição (ANVISA, 2010). Foram pesados exatamente cerca de 50,00 mg de pirogalol e dissolvidos em balão volumétrico de $100 \mathrm{~mL}$, com água destilada. Alíquota de 5,0 mL dessa solução foi volumetricamente transferida para balão volumétrico de $100 \mathrm{~mL}$ e o volume, completado com água destilada. Em balão volumétrico de $25 \mathrm{~mL}$, foram transferidos 2,0 mL dessa solução, 3,0 $\mathrm{mL}$ do reagente Folin-Ciocalteu, $10,0 \mathrm{~mL}$ de água destilada e o volume, completado com solução de carbonato de sódio $\left(\mathrm{Na}_{2} \mathrm{CO}_{3}\right)$ a $10,75 \%(\mathrm{~m} / \mathrm{v})$. A absorvância foi determinada em $760 \mathrm{~nm}$ após $30 \mathrm{~min}$, utilizando-se água destilada como solução de compensação. Todas as operações foram protegidas da luz.

\subsubsection{Cálculo do Teor de Taninos Totais}

O teor de taninos totais foi determinado pela seguinte equação:

$$
T T=\frac{6,25 \times(A 1-A 2) \times M 2}{A 3 \times M 1}
$$

em que TT representa o teor de taninos totais; A 1 e A2 consistem, respectivamente, na absorvância da amostra para polifenóis totais e na absorvância da amostra para polifenóis não adsorvidos em pó de pele; $\mathrm{M}_{1}$ é a massa da amostra utilizada no ensaio, em gramas, considerando-se a perda por dessecação; $M_{2}$ é a massa de pirogalol, em gramas; e A3 é a absorvância da soluçãopadrão.

\subsection{Desenvolvimento do Método}

\subsubsection{Quantidade de Droga e Volume do Reagente Folin- Ciocaulteu}

Os efeitos da quantidade de droga $(0,5000 ; 1,0000$ e $1,5000 \mathrm{~g})$ e volume do reagente Folin-Ciocalteu (1,0, 2,0 e $3,0 \mathrm{~mL}$ ) sobre a resposta do método foram estudados

Revista Árvore, Viçosa-MG, v.39, n.1,p.37-47, 2015 
através de um experimento fatorial do tipo $3^{2}$ adicionado de dois pontos centrais $(1,0000 \mathrm{~g}$ de droga e $2,0 \mathrm{~mL}$ de Folin). Cada ponto representou uma média de três determinações.

As variáveis independentes para o desenho experimental incluíram quantidade de droga e volume de reagente. A variável dependente estudada (resposta) foi a absorvância. A matriz experimental está representada na Tabela 1, sendo a reposta estabelecida por um modelo de segunda ordem. A análise estatística do modelo foi realizada através do método dos mínimos quadrados e o modelo matemático obtido, usado para gerar superfícieresposta com o auxílio do programa computacional STATISTICA 6.0 (StatSoft, USA).

\subsubsection{Concentração de Carbonato de Sódio $\left(\mathrm{Na}_{2} \mathrm{CO}_{3}\right)$}

Após a determinação da quantidade ideal de droga e do volume adequado do reagente, foi avaliada a concentração ótima de carbonato de sódio, comparandose soluções distintas de 10,$75 ; 14$; e $29 \%$, m/v. A absorvância foi determinada e o teor de taninos totais, expresso como média de três determinações.

\subsubsection{Agente Precipitante}

Por fim, foi avaliado o melhor agente precipitante, cujo critério de escolha incluiu a maior absorvância, bem como a quantidade ideal do referido agente. Foram comparados: caseína $(150,0,250,0$ e $350,0 \mathrm{mg})$, polivinilpirrolidona $(50,00100,0$ e $200,0 \mathrm{mg})$ e pó de pele $(50,00100,0$ e $150,0 \mathrm{mg})$. A absorvância foi determinada e o teor de taninos totais, expresso como média de três determinações.

\subsection{Validação}

Os parâmetros de validação foram analisados de acordo com os requisitos preconizados pelo Guia para Validação de Métodos Analíticos e Bioanalíticos, disponível na RE 899 (BRASIL, 2003), e pelo International Conference on the Harmonization of Technical Requirements for the Registration of Pharmaceuticals for Human Use (ICH, 2005). Todos os ensaios de validação foram realizados nas condições espectrofotométricas estabelecidas após o estudo de desenvolvimento.

Os resultados foram apresentados como média \pm erro-padrão da média. Também, foram utilizados análise de variância (ANOVA) e teste $t$, de Student, quando pertinentes, com o auxílio do software Excel (Microsoft Corporation, USA). O limite de significância foi considerado como $5 \%(\mathrm{p}<0,05)$.

\subsubsection{Linearidade da Amostra e do Padrão}

A linearidade da amostra e do padrão foi avaliada através da análise de regressão linear pelo método dos mínimos quadrados, a partir de três curvas de calibração autênticas, usando-se cinco concentrações. Os resultados permitiram definir o coeficiente de determinação (mínimo aceitável $\mathrm{R}^{2}=0,99$ ), o desvio-padrão relativo (DPR) e a equação de regressão. Também foram efetuados testes de falta de ajuste do modelo linear e significância da regressão (PIMENTEL; BARROS, 1996).

Tabela 1 - Matriz do planejamento fatorial aplicada para avaliar a influência da quantidade de droga e volume do reagente Folin-Ciocaulteu na resposta do método (absorvância).

Table 1 - Matrix of factorial design applied to evaluate the influence of drug amount and Folin-Ciocaulteu reagent volume on the method response (absorbance).

\begin{tabular}{|c|c|c|c|c|c|}
\hline \multirow{2}{*}{ Experimento } & \multicolumn{2}{|c|}{ Variáveis codificadas } & \multicolumn{2}{|c|}{ Variáveis naturais } & \multirow{2}{*}{ Resposta do método } \\
\hline & QD (g) & $\mathrm{VR}(\mathrm{mL})$ & QD (g) & $\mathrm{VR}(\mathrm{mL})$ & \\
\hline 1 & -1 & -1 & 0,5 & 1 & 0,325 \\
\hline 2 & -1 & +1 & 0,5 & 3 & 0,333 \\
\hline 3 & +1 & -1 & 1,5 & 1 & 0,598 \\
\hline 4 & +1 & +1 & 1,5 & 3 & 0,741 \\
\hline 5 & 0 & 0 & 1,0 & 2 & 0,516 \\
\hline 6 & 0 & 0 & 1,0 & 2 & 0,524 \\
\hline 7 & 0 & 0 & 1,0 & 2 & 0,532 \\
\hline 8 & 0 & -1 & 1,0 & 1 & 0,411 \\
\hline 9 & 0 & +1 & 1,0 & 3 & 0,514 \\
\hline 10 & -1 & 0 & 0,5 & 2 & 0,262 \\
\hline 11 & +1 & 0 & 1,5 & 2 & 0,634 \\
\hline
\end{tabular}

Revista Árvore, Viçosa-MG, v.39, n.1, p.37-47, 2015 
As faixas de concentração utilizadas para o pirogalol e para a amostra foram, respectivamente, de 1 a $5 \mu \mathrm{g} /$ $\mathrm{mL}$ e de 6 a $30 \mu \mathrm{g} / \mathrm{mL}$.

\subsubsection{Limites de Detecção e de Quantificação}

Os limites de detecção (LD) e de quantificação (LQ) foram estimados (em $\mu \mathrm{g} / \mathrm{mL}$ ) considerando o desviopadrão (DP) com relação à absorvância da menor concentração e ao coeficiente angular (inclinação da reta-IC), obtidos pela linearidade do padrão e da amostra (equações 2 e 3 ).

$$
\begin{aligned}
& L D=\frac{D P \times 3}{I C} \\
& L Q=\frac{D P \times 10}{I C}
\end{aligned}
$$

\subsubsection{Especificidade}

A especificidade foi avaliada pelo método de adição de padrão, no qual foi adicionado $1,0 \mathrm{~mL}$ de pirogalol $(1 \mu \mathrm{g} / \mathrm{mL})$ em cada uma das cinco concentrações utilizadas no teste de linearidade. O método foi considerado específico quando os coeficientes angulares das equações da reta de linearidade e especificidade foram considerados semelhantes (BLAINSKI et al., 2013).

\subsubsection{Precisão}

A precisão da metodologia espectrofotométrica foi avaliada nos níveis de repetibilidade e precisão intermediária. A repetibilidade foi definida pela análise de seis amostras individuais da solução extrativa a $100 \%$ de sua concentração $(18 \mu \mathrm{g} / \mathrm{mL})$. A precisão intermediária foi avaliada em triplicata, em dois dias consecutivos, por dois analistas diferentes. A repetibilidade foi expressa através do desvio-padrão relativo (DPR) e a precisão intermediária, avaliada através de ANOVA, além do DPR (RIBANI et al., 2004).

\subsubsection{Exatidão}

A recuperação foi calculada pela adição de quantidades conhecidas e crescentes da solução-padrão de pirogalol ( 1 a $3 \mu \mathrm{g} / \mathrm{mL}$ ), em amostras na concentração de análise de $100 \%$. Os testes foram realizados em triplicata para cada nível de concentração, com ensaios realizados em triplicata, sendo calculado o percentual de recuperação. O método foi considerado exato quando o percentual de recuperação atingiu a faixa entre 85 e $115 \%$ (BRASIL, 2003; BLAINSKI et al., 2013).

\subsubsection{Robustez}

A robustez do método foi determinada através de variações na luminosidade (presença ou ausência de luz) e no comprimento de onda (758, 760 e $762 \mathrm{~nm})$. As análises foram realizadas em triplicata, utilizando-se a solução extrativa a $100 \%$ de sua concentração. A análise dos resultados foi feita através de ANOVA OneWay.

\section{RESULTADOS}

\subsection{Desenvolvimento do Método}

\subsubsection{Quantidade de Droga e Volume do Reagente Folin-Ciocaulteu}

Os resultados do desempenho do método com diferentes quantidades de droga e, ou, volume de reagente Folin-Ciocalteu encontram-se na Tabela 1. Os dados experimentais foram usados para gerar modelos de segunda ordem para cada variável dependente. Visto que o teste de falta de ajuste não foi significante e as variações experimentais puderam ser atribuídas apenas ao erro aleatório. Diante disso, os modelos ajustados demonstraram aproximação adequada dos valores reais e propiciaram a construção do gráfico superfície-resposta. A análise desse gráfico evidenciou que há influência de ambas as variáveis analisadas: com o aumento do volume do reagente Folin-Ciocalteu, verificou-se aumento na resposta do método, mas esse aumento é muito mais pronunciado quando se eleva a quantidade de droga (Figura 1).

AANOVA foi significativa para ambas as variáveis, quantidade de droga e volume de reagente, demonstrando que sua variação influencia significativamente a resposta estudada. O gráfico de Pareto, construído a partir do teste $t$, de Student, revelou importância estatística para os termos lineares de ambas as variáveis independentes, principalmente quantidade de droga. Entre os termos quadráticos, apenas a quantidade de droga foi estatisticamente significativa (Figura 2).

\subsubsection{Concentração de Carbonato de Sódio $\left(\mathrm{Na}_{2} \mathrm{CO}_{3}\right)$ e Agente Precipitante}

A mudança nas concentrações de carbonato de sódio levou em consideração dados da literatura: 7,5\% (BLAINSKI et al., 2013), 10,75\% (GLASL, 1983) e 14\% (EUROPE COUNCIL, 2007). A avaliação das três concentrações distintas de carbonato de sódio não revelou

Revista Árvore, Viçosa-MG, v.39, n.1,p.37-47, 2015 
nenhuma diferença estatisticamente significativa entre as variações estabelecidas. No que se refere ao agente precipitante, o pó de pele demonstrou melhores resultados

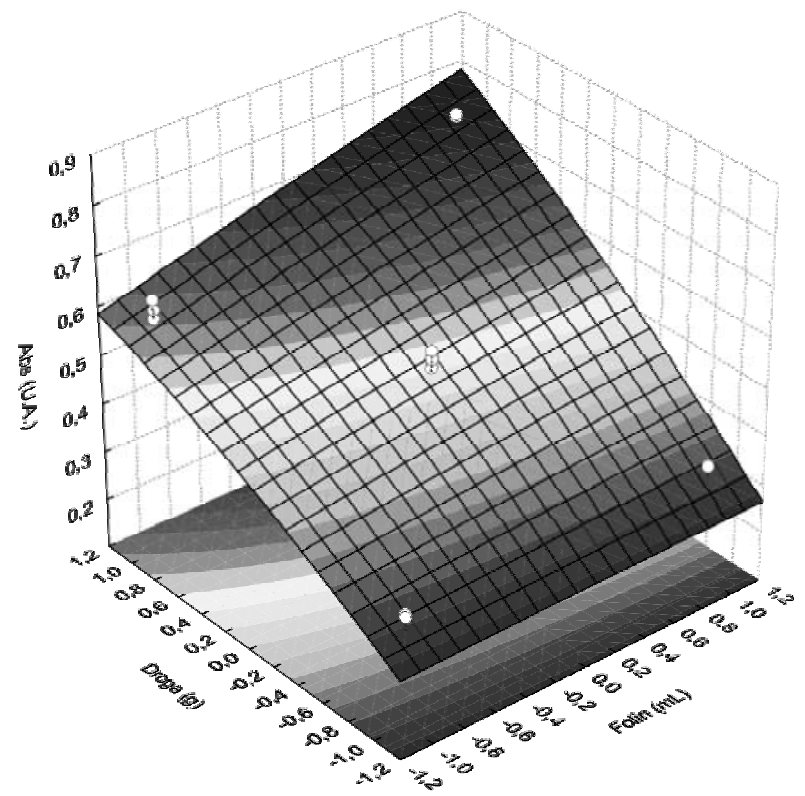

Figura 1 - Superfície de resposta obtida através da análise fatorial em valores de absorvância (UA).

Figure 1 - Response surface obtained by factorial analysis in absorbance values (AU).

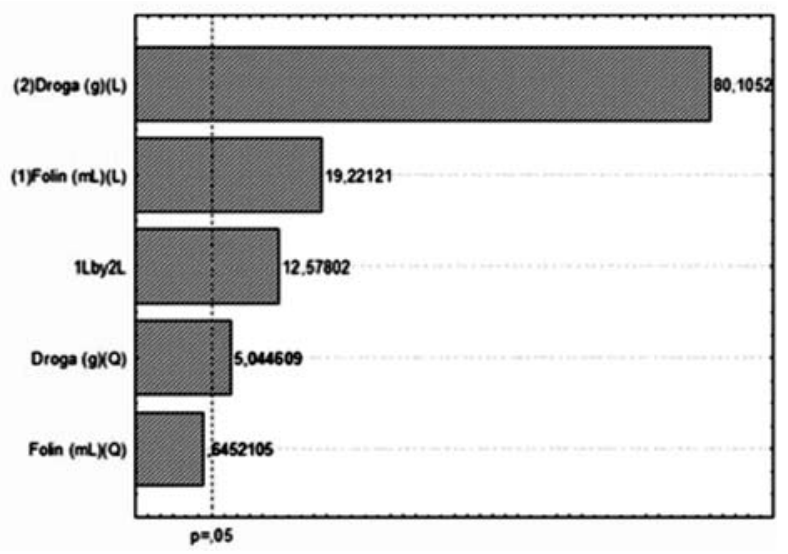

Figura 2 - Gráfico de Pareto para as variáveis: quantidade de droga e volume de Folin-Ciocalteu. $\mathrm{L}=$ termo linear; $\mathrm{Q}=$ termo quadrático; e $1 \mathrm{Lby} 2 \mathrm{~L}=$ interação volume de Folin $\mathrm{x}$ quantidade de droga.

Figure 2 - Pareto graph for the variables: drug amount and Folin-Ciocalteu volume. $L=$ linear term; $Q=$ quadratic term; and 1 Lby $2 L=$ interaction Folin volume x drug amount. para determinação do teor de taninos totais quando comparado com os demais. Além disso, aumento na sua quantidade resultou em incremento da resposta ao método.

\subsection{Validação}

\subsubsection{Linearidade e Limites de Detecção (LD) e Quantificação (LQ)}

As curvas de calibração do padrão e taninos totais nas cascas de S. amara por espectrofotometria apresentaram linearidade dentro do intervalo de 80 a $120 \%$, na faixa de concentração estabelecida e no comprimento de onda de $760 \mathrm{~nm}$ (Tabela 2). A análise de regressão linear indicou coeficientes de determinação de 0,9992 e 0,9982, respectivamente, para a curva-padrão e de taninos, valores que comprovam que mais de $99 \%$ da variabilidade experimental pode ser explicada pelos modelos lineares e que há adequação do método no que concerne à relação entre as concentrações do analito e as respostas espectrofotométricas.

Os limites de detecção e quantificação, também descritos na Tabela 2, foram determinados utilizandose o desvio-padrão médio do primeiro ponto da curva de calibração do pirogalol (equações 2 e 3 ).

\subsubsection{Especificidade}

A especificidade de métodos espectrofotométricos para matrizes complexas, como matérias-primas vegetais, é de fundamental importância para garantir confiabilidade na quantificação de metabólitos secundários (LANDIN et al., 2012). No caso de matrizes complexas, se a matriz sem o analito não está disponível, os efeitos do sistema matricial podem ser analisados, comparando-se os coeficientes angulares das curvas de linearidade e especificidade. Se as curvas forem paralelas, o método pode ser considerado específico (BLAINSKI et al., 2013). A especificidade do método para a solução extrativa foi confirmada, uma vez que os coeficientes angulares das equações lineares foram muito próximos (Figura 3 ).

\subsubsection{Precisão}

Os resultados evidenciaram que, em relação à repetibilidade, o método corrobora os parâmetros exigidos pela legislação vigente, apresentando desviopadrão relativo (DPR) inferior a 5\%.

Quanto à precisão intermediária, além do DPR inferior a 5\%, a análise estatística demonstrou que o $\mathrm{F}$ calculado $(3,45)$ foi menor do que o F tabelado $(4,07)$, o que comprova

Revista Árvore, Viçosa-MG, v.39, n.1, p.37-47, 2015

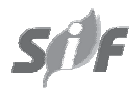


Tabela 2 - Curvas de calibração do padrão (pirogalol) e extrato da casca do caule de Simarouba amara. Table 2 - Calibration curves of the standard (pyrogallol) and stem bark extract of Simarouba amara.

\begin{tabular}{|c|c|c|c|c|c|c|}
\hline Amostra & $\begin{array}{l}\text { Coeficientes } \\
\text { angular e linear }\end{array}$ & Faixa $(\mu \mathrm{g} / \mathrm{mL})$ & LD e LQ & $\mathrm{R}^{2}$ & Significância da regressão & Falta de ajuste \\
\hline Pirogalol & $\begin{array}{l}a=0,1709 \\
b=0,0493\end{array}$ & $1,0-5,0$ & $\begin{array}{l}\mathrm{LD}=0,0709 \\
\mathrm{LQ}=0,2365\end{array}$ & 0,9992 & $\begin{array}{c}\text { F Calculado: } \\
63,94 \\
\text { F Tabelado: } \\
4,557\end{array}$ & $\begin{array}{c}\text { F Calculado: } \\
0,005 \\
\text { F Tabelado: } \\
3,708\end{array}$ \\
\hline Simarouba amara & $\begin{array}{l}a=0,1327 \\
b=0,1795\end{array}$ & $6,0-30,0$ & $\begin{array}{l}\mathrm{LD}=0,0977 \\
\mathrm{LQ}=0,3258\end{array}$ & 0,9982 & $\begin{array}{c}\text { F Calculado: } \\
31,59 \\
\text { F Tabelado: } \\
4,557\end{array}$ & $\begin{array}{c}\text { F Calculado: } \\
\text { 3,31 } \\
\text { F Tabelado: } \\
3,708\end{array}$ \\
\hline
\end{tabular}

LD = Limite de Detecção; LQ = Limite de Quantificação; e R²= Coeficiente de Determinação. $D L=$ Detection Limit; $Q L=$ Quantification Limit; and R2 = Determination Coefficient.

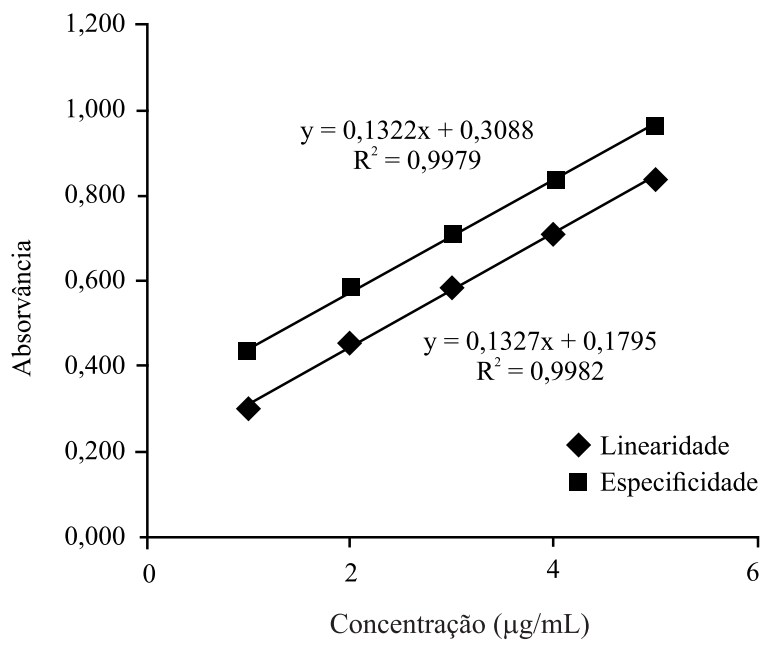

Figura 3 - Curvas de linearidade e especificidade da solução extrativa de Simarouba amara.

Figure 3-Linearity and specificity curves of extractive solution of Simarouba amara.

que não há diferença estatisticamente significativa entre as amostras analisadas em dias distintos por diferentes analistas, no nível de 95\% de confiança.

\subsubsection{Exatidão}

Para as concentrações baixa, média e alta, os resultados evidenciaram percentuais de recuperação iguais a 92,01; 102,21; e 109,33\%, respectivamente. Esses percentuais encontram-se dentro da faixa de $85-115 \%$, preconizada pela legislação vigente (BRASIL, 2003). O DPR foi inferior a $5 \%$ em todos os níveis de concentração avaliados, o que demonstra a exatidão do método e a confiabilidade dos resultados.

\subsubsection{Robustez}

Para avaliação da robustez do método, foram realizadas pequenas e deliberadas variações nos parâmetros de presença/ausência de luz e comprimento de onda. A luminosidade foi estudada quanto à proteção ou não das amostras contra a luz, desde a adição do reagente Folin-Ciocalteu até o momento de leitura no espectrofotômetro. Já o comprimento de onda foi avaliado nos níveis de 758, 760 e $762 \mathrm{~nm}$. Todos os resultados apresentaram coeficiente de variação inferior a 5\%, corroborando a RE 899, e não exibiram diferença estatisticamente significativa entre si, no teste $t$, de Student, pois o F calculado foi menor que o $\mathrm{F}$ tabelado, tanto para a variável luminosidade $(\mathrm{F}$ calculado $=2,57 ; \mathrm{F}$ tabelado $=7,729)$ quanto para a variável comprimento de onda ( $\mathrm{F}$ calculado $=0,386$; $\mathrm{F}$ tabelado $=5,143)$. Esses resultados confirmam a robustez do método.

\section{DISCUSSÃO}

No desenvolvimento do método, tanto o gráfico de superfície-resposta, construído a partir da análise fatorial para as variáveis volume do reagente e quantidade de droga vegetal, quanto os ensaios que compararam diferentes concentrações de carbonato de sódio e quantidades distintas de pó de pele permitiram estabelecer parâmetros através dos quais foram alcançadas as condições ótimas para determinação espectrofotométrica de taninos totais em S. amara, pelo método de FolinCiocalteu. Esses valores incluíram: $1,5000 \mathrm{~g}$ de droga vegetal; 3,0 mL do reagente Folin-Ciocalteu; carbonato de sódio $10,75 \%$ e $150,0 \mathrm{mg}$ de pó de pele.

Revista Árvore, Viçosa-MG, v.39, n.1,p.37-47, 2015 
A validação, exigida pela RDC n ${ }^{\circ} 14$ (BRASIL, 2010) e regulamentada pela RE 899, contribuiu para identificar e controlar fatores de variação, estabelecer limites de aceitação do erro analítico e assegurar a confiabilidade dos resultados (BRASIL, 2003).

Os resultados obtidos a partir da linearidade demonstraram que a variável dependente (absorvância) é diretamente proporcional à variável independente (concentração do analito) dentro do intervalo estudado e que a equação matemática obtida expressa adequadamente essa relação. Além disso, os valores expressos na Tabela 1 revelaram que a metodologia espectrofotométrica foi sensível para a determinação de taninos na solução extrativa da casca do caule de S. amara, sem sofrer alterações intrínsecas do equipamento.

A especificidade de um método consiste na sua capacidade de distinguir o analito na presença de outros componentes da matriz, os quais podem incluir impurezas, produtos de degradação, entre outros (ICH, 2005). O paralelismo das retas confirmou que, nas condições experimentais, foi possível quantificar especificamente o padrão pirogalol, assim como os taninos presentes na solução extrativa, mesmo na presença de outros compostos ou impurezas.

A precisão é considerada uma das análises mais representativas, visto que revela o efeito das variações relacionadas aos dias, analistas ou equipamentos distintos, implicando garantia da reprodutibilidade do método (BUENO, 2012). A exatidão, por sua vez, reflete a proximidade dos resultados obtidos por uma metodologia analítica em relação aos valores verdadeiros (BRASIL, 2003). Procedimentos exatos devem estar livres de interferências, como condições extrativas e reagentes semelhantes, e as respostas da metodologia devem estar relacionadas com a concentração do analito (ARAÚJO et al., 2013). Os resultados expressos em ambos os ensaios demonstraram que o método é preciso e exato, o que contribui para a garantia da sua confiabilidade.

O estudo da robustez, definida como a capacidade do método em resistir a pequenas e deliberadas variações de parâmetros analíticos, sem perder sua exatidão e precisão (ICH, 1995), permitiu verificar que a metodologia proposta não sofreu interferências significativas diante de pequenas variações na concentração de carbonato de sódio, presença de luz e comprimento de onda, contribuindo para fornecer indicação de confiança no processo durante seu desenvolvimento usual.

\section{CONCLUSÃO}

A quantificação de metabólitos secundários exerce importante papel no controle de qualidade da matériaprima vegetal. Nesse contexto, uma metodologia espectrofotométrica na faixa do visível para determinação de taninos totais na casca do caule de S. amara foi adequadamente desenvolvida e validada. O método proposto foi linear, específico, exato, preciso e robusto, sob as condições estudadas, e destacou-se pela facilidade de execução (desde o preparo até a leitura das amostras) e baixo custo, estando em conformidade com os requisitos para o uso analítico e para garantir a confiabilidade dos resultados.

\section{AGRADECIMENTOS}

Ao Conselho Nacional de Desenvolvimento Científico e Tecnológico (475216/2011-3; 483870/2011; 0480128/2012-0; 302113/2012-6); e FACEPE (APQ-12964.03/12), pelo suporte financeiro.

\section{REFERÊNCIAS}

ALMEIDA, M. M. B.; ARRIAGA, A. M. C.; SANTOS, A. K. L.; LEMOS, T. L. G.; BRAZFILHO, R.; VIEIRA, I. J. C. Ocorrência e atividade biológica de quassinoides da última década. Quimica Nova, v.30, n.4, p.935-951, 2007.

AMARAL, F. M. M.; RIBEIRO, M. N. S.; BARBOSA-FILHO, J. M.; REIS, A. S.; NASCIMENTO, F. R. F.; MACEDO, R. O. Plants and chemical constituints with giardicidal activity. Revista Brasileira de Farmacognosia, v.16, p.696720, 2006.

\section{AGÊNCIA NACIONAL DE VIGILÂNCIA} SANITÁRIA - ANVISA. Farmacopeia Brasileira. 5.ed. São Paulo: Atheneu, 2010. 2v.

ARAÚJO, L; B. D. C.; SILVA, S. L.; GALVÃO, M. A. M.; FERREIRA, M. R. A.; ARAÚJO, E. L.; RANDAU, K. P.; SOARES, L. A. L. Total phytosterol content in drug materials and extracts from roots of Acanthospermum hispidum by UVVIS spectrophotometry. Revista Brasileira de Farmacognosia, v.23, n.5, p.736-742, 2013.

ARRIAGA, A. M. C.; MESQUITA, A. C.; PULIQUEN, Y. B. M.; LIMA, R. A.; CAVALCANTE, S. H.; CARVALHO, M. G.; 
SIQUEIRA, J. A.; ALEGRIO, L. V.; BRAZ-FILHO, R. Chemical constituents of Simarouba versicolor. Anais da Academia Brasileira de Ciência, v.74, n.3, p.415-424, 2002.

BLAINSKI, A.; LOPES, G. C.; MELLO, J. C. P. Application and analysis of the Folin-Ciocaulteu method for the determination of the total phenolic content from Limonium brasiliense L. Molecules, v.18, p.6852-6864, 2013.

BRASIL, Agência Nacional de Vigilância Sanitária, Resolução RE N899, de 29 de Maio de 2003.

Diário Oficial [da] República

Federativa do Brasil, Brasília, DF, 02 jun. 2003.

BRASIL, Resolução RDC 26 - Dispõe sobre o registro de medicamentos fitoterápicos e o registro e a notificação de produtos tradicionais fitoterápicos . ANVISA, Diário Oficial [da] República Federativa do Brasil. Brasília, DF, maio. 2014.

BUENO, F. G.; MACHARETH, M. A. D.; PANIZZON, G. P.; LOPES, G. C.; MELLO, J. C. P.; LEITE-MELLO, E. V. S. Development of a uv/vis spectrophotometric method for analysis of total polyphenols from Caesalpinia peltophoroides Benth. Quimica Nova, v.35, n.4, p.822-826, 2012.

CACERES, A.; CANO, O.; SAMAYOA, B.; AQUILAR, L. Plants used in Guatemala for the treatment of gastrointestinal disorders. 1. Screening of 84 plants against enterobacteria. Journal of Ethnopharmacology, v.30, n.1, p.55-73, 1990.

CACHET, N.; HOAKWIE, F.; BERTANI, S.; BOURDY, G.; DEHARO, E.; STIEN, D.; HOUEL, E.; GORNITZA, H.; FILLAUX, J.; CHEVALLEY, S.; VALENTIN, A.; JULLIAN, V. Antimalarial activity of simalikalactone $\mathrm{E}$, a new quassinoid from Quassia amara L. (Simaroubaceae).

Antimicrobial

Agentsand Chemotherapy, v.53, n.10, p.4393-4398, 2009.

DECLERCQ, J. Suspected wood poisoning caused by Simarouba amara (marupá/caixeta) shavings in two dogs with erosive stomatitis and dermatitis. Veterinary Dermatology, v. 15, p.188-193, 2004.
EUROPE COUNCIL. Determination of tannins in herbal drugs. In: European

Pharmacopoeia. 6.ed. Strasbourg: European Directorate for the Quality of Medicines, 2007. p.A286.

FERNANDES, A. J.; FERREIRA, M. R.; RANDAU, K. P.; SOUZA, T. P.; SOARES, L. A. Total flavonoids content in the raw material and aqueous extractives from Bauhinia monandra Kurz (Caesalpiniaceae). The Scientific World Journal, v.2012, p.1-7, 2012.

FOLIN, O.; CIOCALTEU, V. On tyrosine and tryptophan determination proteins. The Journal of Biological Chemistry, v.73, n.2, p.627, 650, 1927.

GALLICE, W. C.; MESSERSCHIMIDT, I.; PERALTA-ZAMORA, P. Caracterização espectroscópica multivariada do potencial antioxidante de vinhos. Quimica Nova, v.34, n.3, p.397-403, 2011.

GOSH, P. C.; LARRAHONDO, J. E.; LEQUESNE, P. W.; RAFFAUF, R. F. Antitumor plants. IV. Constituents of Simarouba versicolor. Lloydia, v.40, n.4, p.364-369, 1977.

GLASL, H. Zur Photometrie in der drogenstandardisierung. 3. Gehaltsbestimmung von gerbstoffdrogen. Deutscher Apotheker Zeitung, v.123, p.1979-1983, 1983.

GOVINDARAJU, K.; DARUKESHWARA, J.; SRIVASTAVA, A. K. Studies on protein characteristics and toxic constituents of Simarouba glauca oilseed meal. Food and Chemical Toxicology, v.47, n.6, p.1327-1332, 2009.

GROSVENOR, N. J.; MASCOLL, K.; MCLEAN, S.; REYNOLDS, W. F.; TINTO, W. F. Tirucallane, apotirucallane, and octanorapotirucallane triterpenes of Simarouba amara. Journal National

Production, v. 69, n.6, p. 1315-1318, 2006.

ICH. International Conference on Harmonization of Technical Requirements for Registration of Pharmaceuticals for Human Use. In: Q2B(R1): Guideline on validation of analytical procedure- methodology. Brussels: $\mathrm{ICH}$ Steering Committee, 2005.

Revista Árvore, Viçosa-MG, v.39, n.1,p.37-47, 2015 
KAIJ-A-KAMB, M.; AMOROS, M.; GIRRE, L. Search for new antiviral agents of plant origin. Pharmaceutica Acta Helvetica, v.67, n.5/ 6, p.130-147, 1992.

KAUR, K. Antimalarials from nature. Bioorganic \& Medicinal Chemistry, v.17, p.3229-3256, 2009.

LANDIN, L. P.; FEITOZA, G. S.; COSTA. J. G. M. Development and validation of a HPLC method for the quantification of three flavonoids in a crude extract of Dimorphandra gardneriana. Revista Brasileira de Farmacognosia, v.23, n.1, p.58-64, 2012.

LASSAK, E. V.; POLONSKY, J.; JACQUEMIN, H. 5-Hydroxiy-canthin-6-one from Simarouba amara. Phytochemistry, v. 16, p.1126-1127, 1977.

LOPEZ, C. G.; SANCHES, C. C. A.; NAKAMURA, C. V. Influence of extracts of Stryphnodendron polyphyllum Marth and Stryphnodendron obovatum Benth. On the cicatrization of cutaneous wounds in rats. Journal of Etnopharmacology, v.99, n.2, p.265-272, 2005.

LORENZI, H. Árvores brasileiras: manual de identificação e cultivo de plantas arbóreas nativas do Brasil. 5.ed. Nova Odessa: Plantarum, 2002.324p.

LORENZI, H.; MATOS, F. J. A. Plantas medicinais no Brasil: nativas e exóticas. Nova Odessa: Plantarum, 2002. 512p.

LOUREIRO, A. A.; SILVA, M. F.; ALENCAR, J. C. Essências madeireiras da Amazônia. Manaus: INPA, 1979. 432p.

MARIATH, I. R.; FALCÃO, H. S.; BARBOSAFILHO, J. M.; SOUSA, L. C. F.; TOMAZ, A. C. A.; BATISTA, L. M.; DINIZ, M. F. F. M.; ATAHYDEFILHO, P. F.; TAVARES, J. F.; SILVA, M. S.; CUNHA, E. V. L. Plants of the American continent with antimalarial activity. Revista Brasileira Farmacognosia, v. 19, n.1A, p.158-192, 2009.

MARQUES, G. S.; LEÃO, W. F.; LYRA, M. A. M.; PEIXOTO, M. S.; MONTEIRO, R. P. M.; ROLIM, L. A.; XAVIER, H. S.; NETO, P. J. R.; SOARES, L. A. L. Comparative evaluation of UV/VIS and HPLC analyticalmethodologies applied for

Revista Árvore, Viçosa-MG, v.39, n.1, p.37-47, 2015 quantification of flavonoids from leaves of Bauhinia forficata. Revista Brasileira Farmacognosia, v.23, n.1, p.51-57, 2013.

MONTEIRO, J. M. M.; NETO, E. M. F. L.; AMORIM, E. L. C.; STRATTMANN, R. R.; ARAÚJO, E. L.; ALBUQUERQUE, U. P. Teor de taninos em três espécies medicinais arbóreas simpátricas da caatinga. Revista Árvore, v.29, n.6, p.999-1005, 2005.

MUHAMMAD, I.; BEDIR, E.; KHAN, S. I.; TEKWANI, B. L.; KHAN, I. A.; TAKAMATSU, S.; PELLETIER, J.; WALKER, L. A. A new antimalarial quassinoid from Simaba orinocensis. Journal of Natural Products, v.62, p.772-777, 2004.

NERI, V. A.; SOARES, M. P.; MEIRA NETO, J. A. A.; DIAS, L. E. Espécies de cerrado com potencial para recuperação de areas degradadas por mineração de ouro, Paracatu-MG. Revista Árvore, v.35, n.4, p.907-918, 2011.

OGURA, M.; CORDELL, G. A.; KINGHORN, A. D.; FARNSWORTH, N. R. Potential anticancer agents VI.Constituents of Ailanthus excelsa (Simaroubaceae). Lloydia, v.40, n.6, p.579-84, 1977.

PIMENTEL, M. F.; BARROS, N. B. Calibração: uma revisão para químicos analíticos. Quimica Nova, v.19, n.3, p.268-277, 1996.

PIRANI, J. R.; THOMAS, W.

W. Simaroubaceae in Lista de Espécies da Flora do Brasil. Jardim Botânico do Rio de Janeiro. Disponível em: <http://floradobrasil.jbrj.gov.br/jabot/ floradobrasil/FB1381>. Acesso em: 11 dez. 2013.

POLONSKY, J.; VARON, Z.; JACQUEMIN, H.; PETTIT, G. R. The isolation and structure of 13,18-dehydroglaucarubinone a new antineoplastic quassinoid from Simarouba amara.

Experientia, v.34, n.9, p.1122-1123, 1978.

RIBANI, M.; BOTTOLI, C. B. G.; COLLINS, C. H.; JARDIM, I. C. S. F.; MELO, L. F. C. Validação em métodos cromatográficos e eletroforéticos. Quimica Nova, v.27, n.5, p.771-780, 2004.

RIBEIRO, A. S. S.; PALHA, M. D. C.; TOURINHO, M. M.; WHITEMAN, C. W.; SILVA, A. S. L. Utilização de recursos naturais por comunidades humanas do parque ecoturístico do Guamá, Belém, Pará. Acta Amazonica, v.37, n.2, p.235-240, 2007. 
SARAIVA, R. C. G.; PINTO, A. C. Triterpenos e alcaloide tipo cantinona dos galhos de Simaba polyphylla(Cavalcante) W. W. Thomas (Simaroubaceae). Quimica Nova, v.29, n.2, p.264-268, 2006.

SCOLES, R. Sabiduria popular y plantas medicinales: elejemplo de lacomunidad negra de Itacoã, Acará, Pará. Boletim do Museu. Pararanaense Emílio Goeldi. Ciências Naturais, v.1, n.2, p.79-102, 2006.

SILVA, M. A. B.; MELO, L. V. L.; RIBEIRO, R. V.; SOUZA, J. P. M.; LIMA, J. C. S.; MARTINS, D. T. O.; SILVA, R. M. Levantamento etnobotânico de plantas utilizadas como anti-hiperlipidêmicas e anorexígenas pela população de Nova XavantinaMT, Brasil. Revista Brasileira

Farmacognosia, v.20, n.4, p.549-562, 2010.
SILVA, J. M.; FRANCO, E. S. Florística de espécies arbórea-arbustivas do sub-bosque com potencial fitoterápico em fragmento florestal urbano no município de Camaragibe, Pernambuco, Brasil. Caminhos da Geografia, v.11, n.35, p.179-194, 2010.

VALERIOTE, F. A.; CORBETT, T. H.; GRIECO, P. A.; MOHER, E. D.; COLLINS, J. L.; FLECK, T. J. Anticancer activity of Glaucarubinone analogues. Oncology Research, v. 10, n. 4, p. 201-208, 1998.

WRIGHT, C. W.; O'NEILL, M. J.; PHILLIPSON, J. D.; WARHURST, D. C. Use of microdilution to assess in vitro antiamoebic activities of Brucea javanica fruits, Simarouba amara stem, and a number of quassinoids. Antimicrobial Agents andChemotherapy, v.32, n.11, p.1725-1729, 1988. 